\title{
Using a Planar Array of MEMS Microphones to Obtain Acoustic Images of a Fan Matrix
}

\author{
Lara del Val, ${ }^{1}$ Alberto Izquierdo, ${ }^{2}$ Juan José Villacorta, ${ }^{2}$ and Luis Suárez \\ ${ }^{1}$ Mechanical Engineering Area, Industrial Engineering School, University of Valladolid, Valladolid, Spain \\ ${ }^{2}$ Signal Theory and Communications Department, University of Valladolid, Valladolid, Spain \\ ${ }^{3}$ Civil Engineering Department, Superior Technical College, University of Burgos, Burgos, Spain
}

Correspondence should be addressed to Lara del Val; lvalpue@eii.uva.es

Received 12 March 2017; Accepted 10 August 2017; Published 11 October 2017

Academic Editor: Guiyun Tian

Copyright (C) 2017 Lara del Val et al. This is an open access article distributed under the Creative Commons Attribution License, which permits unrestricted use, distribution, and reproduction in any medium, provided the original work is properly cited.

\begin{abstract}
This paper proposes the use of a signal acquisition and processing system based on an $8 \times 8$ planar array of MEMS (Microelectromechanical Systems) microphones to obtain acoustic images of a fan matrix. A $3 \times 3$ matrix of PC fans has been implemented to perform the study. Some tests to obtain the acoustic images of the individual fans and of the whole matrix have been defined and have been carried out inside an anechoic chamber. The nonstationary signals received by each MEMS microphone and their corresponding spectra have been analyzed, as well as the corresponding acoustic images. The analysis of the acoustic signals spectra reveals the resonance frequency of the individual fans. The obtained results reveal the feasibility of the proposed system to obtained acoustic images of a fan matrix and of its individual fans, in this last case, in order to estimate the real position of the fan inside the matrix.
\end{abstract}

\section{Introduction}

An array is an arranged set of identical sensors, fed in a specific manner. The beam pattern of the array can be controlled by modifying the geometry of the array (linear, planar, etc.), the sensor spacing and the beam pattern, the amplitude, and phase excitation of each sensor [1]. Microphone arrays are a particular case, used in applications such as speech processing, echo cancellation, localization, and sound sources separation [2]. By using beamforming techniques [3], the array beam pattern can be electronically steered to different spatial positions, allowing the discrimination of acoustic sources based on their position.

The authors of this paper have experience in the design [4] and development of acoustic arrays used in many different fields, such as detection and tracking systems $[5,6]$, ambient assisted living [7], or biometric identification systems [8-10]. The arrays used were ULA (Uniform Linear Array), formed by acoustic sensors distributed uniformly along a line. These arrays are simple but limited to one dimension (azimuth or elevation) to estimate the spatial position of the sound source. In order to obtain spatial information in two dimensions, it is necessary to work with planar arrays with sensors distributed on a surface. This is the reason why the authors have developed a new system based on a planar array [11].

Working with planar arrays leads to an increase in both system complexity and space required by the acoustic sensors and the associated hardware, since the extension from a 1D to a $2 \mathrm{D}$ array increases exponentially the number of channels required. These factors are accentuated when the number of sensors to work with is large, despite increasing the number of sensors of the array and also its dimensions, improves the array spatial resolution, because the main lobe width is reduced.

Digital MEMS (Microelectromechanical System) microphones include a microphone, a signal conditioner, and an acquisition device incorporated in the chip itself. For this reason, an acquisition and processing system for an acoustic array based on MEMS microphones is reduced to two basic elements: MEMS microphone and a processing system usually based on an FPGA (Field Programmable Gate Array)

The integration of the microphone preamplifier and the ADC in a single chip significantly reduces costs, if compared with solutions based on analog microphones which require 
four elements: analog microphones with its preamplifier, a signal conditioner, an acquisition device with an analogdigital converter (ADC), memory storage and a digital communication interface, and a signal processor, usually based on a Digital Signal Processor (DSP), which obtains the digitized signals using digital interfaces.

This is one of the main reasons why MEMS microphones are used in acoustic arrays with a large number of channels. But cost reduction is not the only advantage of working with MEMS microphones. This technology also reduces the space occupied by the system, which makes it feasible to build arrays with hundreds or even thousands of sensors.

In recent years techniques for obtaining acoustic images have developed greatly and rapidly. At present, acoustic images are associated with a wide variety of applications, such as nondestructive testing of materials, medical imaging, underwater imaging, SONAR (Sound Navigation And Ranging), and geophysical exploration [12]. Acoustical imaging techniques are based on the RADAR (RAdio Detection And Ranging) principles, forming an image of an object from reflected sound waves instead of radio waves. RADAR systems require high-cost hardware and their application on people and specific materials is difficult, due to the low reflectivity of radio waves. Acoustic images, in comparison with RADAR techniques, represent a simple low cost alternative to RADAR systems.

Arrays of MEMS microphones are specially designed for acoustic source localization [13-15]; however, they are also used in other applications such as DOA (Direction Of Arrival) estimation for vehicles in motion [16], speech processing [17, 18], turbulence measurements [19], identifying geometric dimensions and internal defects of concrete structures [20], or acoustic imaging [21-23]. Due to the high diversity of the applications of these arrays, the authors try to widen these uses to other fields, such as the industrial one. The system used in this paper [11] has two main characteristics/novelties: (i) it is scalable using several subarrays or modules, increasing the number of sensors used, and it is reconfigurable in the position and orientation of the modules and (ii) its multiplatform framework is reconfigurable. These characteristics make the system flexible and suitable for many different applications, such as localization and characterization of noise or vibration sources, spatial filtering and elimination of acoustic interference, acoustic biometry, ambient assisted living, and acoustic holography. This paper presents the use of a system based on a planar array of MEMS microphones to acquire and process acoustic images of a fan matrix.

Fans working at a low speed are less noisy, but they do not move a high amount of air, which is the main objective of a fan. So, what is better in order to move a certain amount of air: having more small fans working at a low speed or fewer big fans working at a higher speed? It is better to have more small fans. Adding two identical noises increases total noise in $3 \mathrm{~dB}$ [24]. In practice, the noises emitted by two are not identical, so the total noise will increase less than $3 \mathrm{~dB}$, because both noises will not be identical. However, doubling the air flow that a fan moves means a high increment in its speed and emitted noise, which supposes a total noise increment higher than $3 \mathrm{~dB}$. The noise emitted by two combined fans will be lower than the noise of a bigger fan moving the same amount of air.

Any type of application that requires specific temperature and humidity conditions is a candidate for a fan matrix. A fan matrix, fan array or fan wall, is a system formed by several fans located on a surface, working together in order to improve the performance of one alone large fan with lower power consumption. Fan matrices are a useful solution for a variety of applications, because of their

(i) installation flexibility,

(ii) operations and maintenance efficiency,

(iii) higher energy efficiency, due to their reduced energy consumption,

(iv) reduced low-frequency noise and minimal vibration levels,

(v) redundancy and reliability features which are not achievable with a conventional fan.

Different applications of fan matrices are mine ventilation $[25,26]$, radiator cooling in vehicles [27-31], ventilation systems [32], airflow control in plant factories [33], air handling in buildings [34-36], or wind tunnels [37], which can be used to simulate tornados [38], hurricanes [39], and tropical storms [40] and also for coastal research [41]. Analyzing these systems it seems that they have no subsystem to control if some of the fans that compose the matrix are down or are not working properly. It would be very useful to detect these kinds of situations. The aim of the authors is to develop a novel fault diagnosis method to detect faulty behaviors of fans included in a matrix. This method will be based on the analysis and classification of acoustic images of the fan matrix, obtained via an array of MEMS microphones, using machine learning techniques. The first step on this research work is to analyze if an array of MEMS microphones could be used to obtain acoustic images of fan matrices.

Section 2 introduces the system used in this study, that is, MEMS microphones which compose the planar array used in the system, as well as the acquisition and processing system used. Section 3 presents the results of using of this system to obtain acoustic images of a fan matrix. Finally, Section 4 contains the conclusions and future research lines.

\section{Materials and Methods}

2.1. MEMS Microphones. The acronym MEMS refers to mechanical systems with a dimension smaller than $1 \mathrm{~mm}$ [42] manufactured with tools and technology arising from the integrated circuits (ICs) field. These systems are mainly used for the miniaturization of mechanical sensors. Their small size makes interconnection with other discrete components more difficult. Therefore, when ordered, they are supplied as part of an encapsulated micromechanical system composed by a sensor, a signal conditioning circuit, and an electric interface [43].

The application of MEMS technology has allowed the development of high-quality microphones with high SNR (Signal to Noise Ratio), low power consumption, and high 


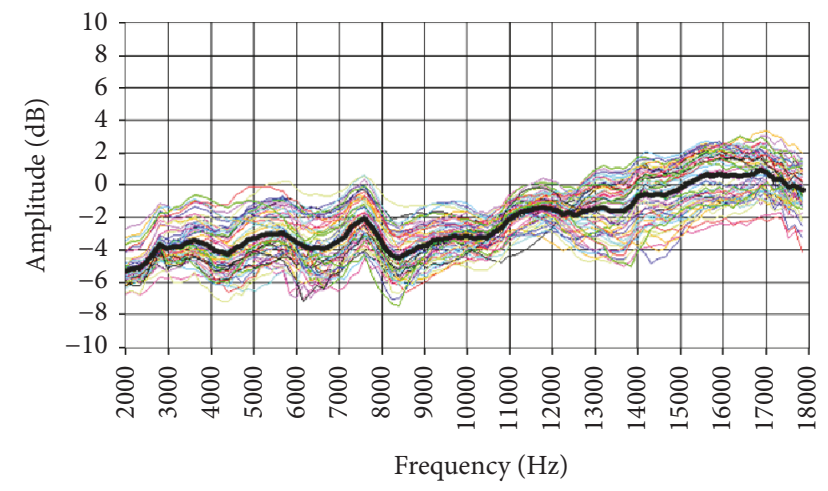

FIGURE 1: Frequency responses and averaged response of the MEMS microphones.

sensitivity. MEMS microphones consist of two components, the acoustic sensor and the controller circuit.

The acoustic sensor usually is a capacitive element formed by two silicon plates: one of them fixed, called backplate, and the other flexible, called diaphragm. Both of them are placed over a chamber on the base of the integrated circuit (wafer). The sound waves move the flexible plate, modifying the capacitance which is measured by the controller circuit [44].

The encapsulation of the MEMS microphones protects the sensor and the controller from external agents. It also allows the sound waves to reach the acoustic sensor, through an opening on the top or on the bottom of the sensor housing. Sensor plates split the internal space into two chambers, which, together with the external opening, yield a Helmholtz resonance effect that produces the amplification of high frequencies. This effect limits the range of frequencies that can be used by the MEMS microphones [45, 46]. Typically, MEMS microphones limit the frequency response to $10 \mathrm{kHz}$; however, with a proper design of the chambers, high-performance MEMS microphones have a flat response up to $20 \mathrm{kHz}$.

For the implementation of the array in which the acoustic system is based, MP34DT01 microphones of STMicroelectronics, digital MEMS microphones with PDM interface [44], were chosen with the following features: low power, omnidirectional response, $63 \mathrm{~dB} \mathrm{SNR}$, high sensitivity (-26 dBFS), and an almost flat frequency response $( \pm 6 \mathrm{~dB}$ in the range from $2 \mathrm{kHz}$ to $16 \mathrm{kHz}$ ).

Figure 1 shows the frequency responses of the MEMS that form the planar array. It can be observed that the averaged frequency response (black thick line) is essentially flat, with a slight increase at high frequencies. This averaged response is bounded within a range of $\pm 4 \mathrm{~dB}$. Figure 1 also shows that the frequency response of MEMS sensors varies in a range of $\pm 2 \mathrm{~dB}$ around the averaged value.

2.2. Processing and Acquisition System. This section shows the acquisition and processing system used in this study, based on a 2D array of MEMS microphones [11]. This system is based on a set of processing routines focused on obtaining acoustic images.

2.2.1. MEMS Array. The acoustic images acquisition system used in this paper is based on a Uniform Planar Array
(UPA) of MEMS microphones. This array, which has been entirely developed by the authors, is a square array of 64 (8 $\times 8)$ MEMS microphones that are spaced uniformly, every $2.125 \mathrm{~cm}$, in a rectangular Printed Circuit Board (PCB), as shown in Figure 2(a). As can also be observed in Figure 2(a), the PCB where the MEMS microphones are placed has square gaps between the acoustic sensors, in order to make the array as light and portable as possible.

This array was designed to work in an acoustic frequency range between 4 and $16 \mathrm{kHz}$. The $2.125 \mathrm{~cm}$ spacing corresponds to $\lambda / 2$ for the $8 \mathrm{kHz}$ frequency. This spacing allows a good resolution for low frequencies, while avoiding grating lobes for high frequencies in the angular exploration zone of interest [8].

2.2.2. Processing System. A myRIO platform [47] is the base unit for this system. This platform belongs to the Reconfigurable Input-Output (RIO) family of devices from National Instruments that is oriented to sensors with nonstandard acquisition procedures, allowing low-level programming of the acquisition routines. The embedded processor included in myRIO is capable of running all the software algorithms to generate acoustic images, so it can be used as a standalone array module formed by a myRIO connected to a MEMS array board as shown in Figure 2(b). The acoustic images can be stored in the internal storage of myRIO or in an external disk connected through the USB port.

Although myRIO can work as a standalone system, the lack of display means that it is usually controlled from a PC (Personal Computer) connected using a Wi-Fi interface. In a global hardware setup, the system includes a PC and one or more array modules. The PC performs three main functions:

(i) As user interface, the PC allows changing the system parameters and visualizing the acoustic images.

(ii) As processing unit, the processors inside the PC, a general purpose PC processor and a Graphical Processing Unit (GPU) could be used to execute the algorithms in order to obtain the acoustic images faster.

(iii) As a control unit, a single PC can manage several myRIO platforms, each one associated with an array module.

2.2.3. Processing Framework. The algorithms implemented in the system [11], shown in Figure 3, can be divided into three blocks: MEMS acquisition, signal processing, and image generation.

The acoustic signals are acquired by the MEMS microphones of the array, and after that they are processed using deinterlacing, decimating, and filtering techniques. Finally, in the image generation block, based on wideband beamforming, a set of $N \times N$ steering directions are defined, and the beamformer output is assessed for each of these steering directions. The images generated are then displayed and stored in the system.

A processing platform enabled to work at a low level, to acquire signals, and at a high level, to implement spatial processing algorithms, using low cost commercial systems, was 


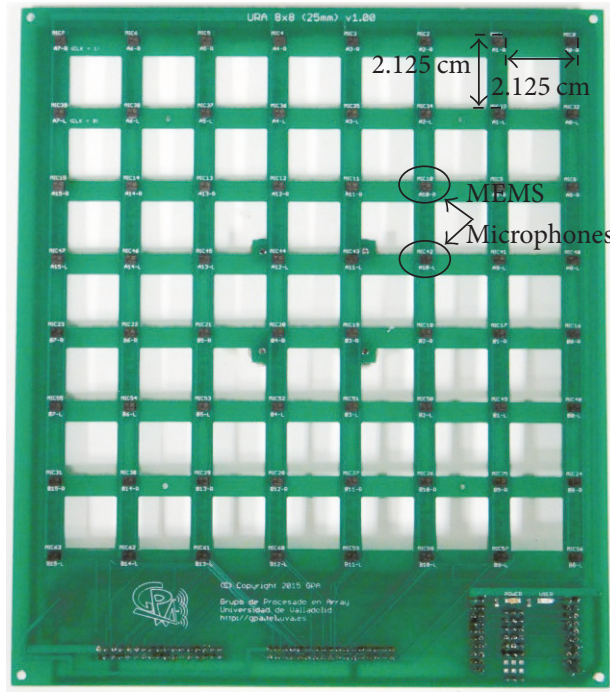

(a)

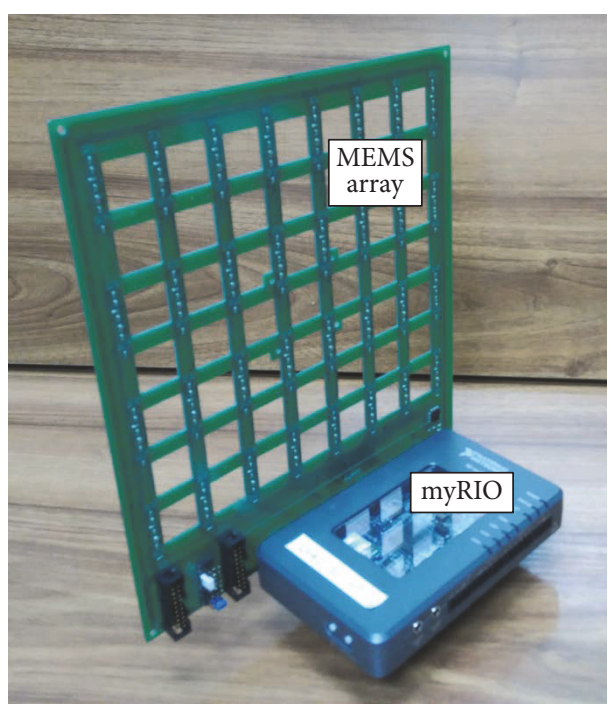

(b)

FIGURE 2: (a) System microphone array. (b) Array module with myRIO and MEMS array board.

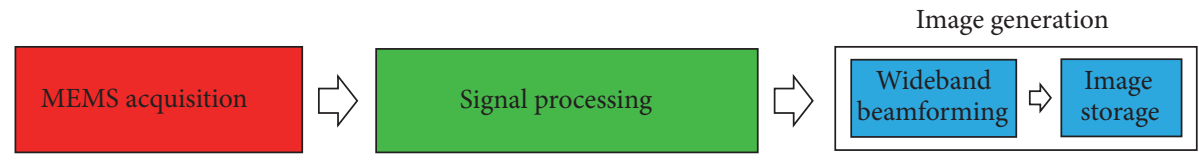

FIgURE 3: Software algorithms diagram.

defined. This processing platform is based on a framework with four processing levels, each one implemented over a general purpose hardware platform:

(i) FPGA: on the basis of its capacity to carry out simple tasks of filtering and decimation

(ii) Embedded processor (EP): an ARM processor that picks up the FPGA signals and carries out the first processing stages

(iii) PC processor: an i5 processor in charge of the main processing of the application

(iv) Graphical Processing Units (GPUs): which can carry out massive FFT (Fast Fourier Transforms) and lineal algebra operations.

The processing algorithms are shared between the FPGA and the PC, excluding beamforming, which is implemented on the GPU, as shown in Figure 4. The embedded processor is used to control and transfer data between the PC and FPGA. This framework is very versatile and is optimal for systems that require a large number of sensors.

2.3. Fan Matrix Tests Assembly. This study case has been carried out with a $3 \times 3$ fan matrix, specifically built to these tests with 9 axial PC fans, which move the air in the direction of the fan axis. Each of the fans used to build the fan matrix is a Foxconn D90SM-12 3-Pin with 7 blades [48]. One of them is shown in Figure 5(a). A PC fan consists of several parts: the engine, the engine control circuitry, the connector, the rotor (the blades), fixed to the engine by means of a bearing system (ball bearings in this specific case), and the frame, with its center fixed to the engine.

Figure 5(b) shows a photograph of the implemented fan matrix. The number of fans in each fan matrix system varies based on airflow requirements. Matrices of nine fans in a three-by-three configuration are a typical arrangement if a redundant configuration is wanted. Normal operations require only eight fans to function, so the ninth fan is redundant. If an active fan fails, the fan matrix system is still totally effective. If two fans fail, the remaining fans can be ramped up [36].

As it can be observed in Figure 5(b), the fans of the matrix are controlled by a Kkmoon 8-channel relay interface board which can be controlled by a microcontroller. In this case, the interface board is controlled by the ARM microcontroller of a myRIO platform. A myRIO platform is used in this control system because it includes an accelerometer that could be useful in the analysis of the acoustic signals obtained with the MEMS microphones of the array, in comparison with the vibrational signals obtained with the accelerometer.

The interface board of the control system allows turning on and off the fans of the matrix independently, by means of software that has been developed with LabVIEW. The user interface of this software shows a $3 \times 3$ matrix of squares, each one representing the fan in the corresponding position. It must be pointed that when a specific fan is running, the corresponding square in the software interface is colored in green; otherwise its color is red. 


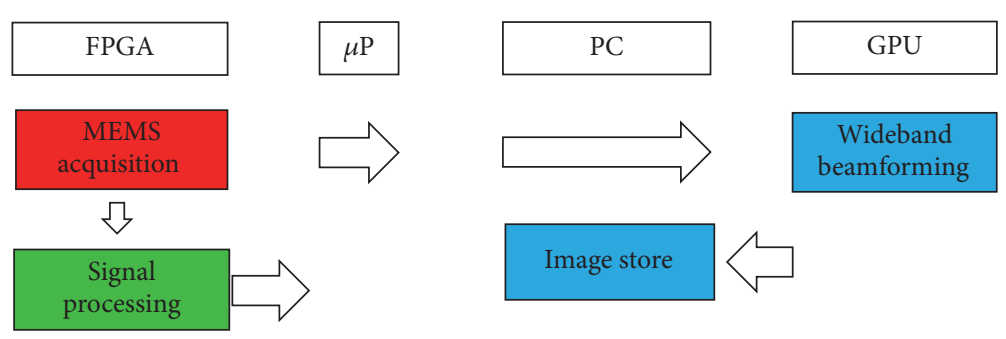

FIGURE 4: System framework.

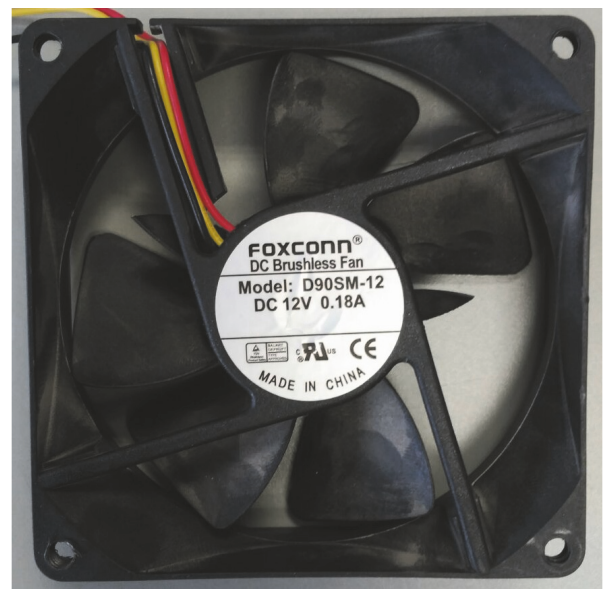

(a)

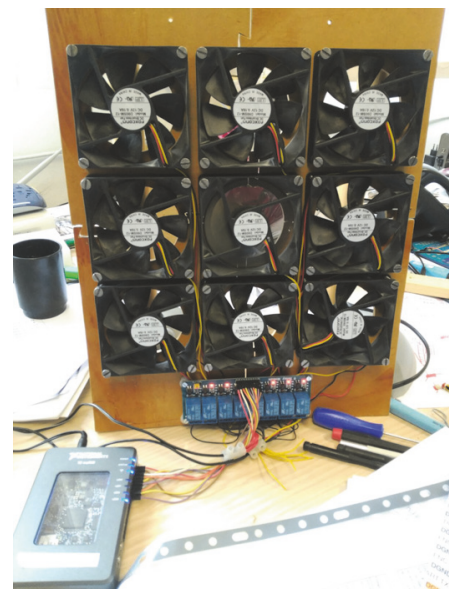

(b)

Figure 5: (a) Foxconn D90SM, 12 fans. (b) Fan matrix built for the test.

This control system has been added to the fan matrix in order to carry out a future work based on fault diagnosis of the matrix by means of the analysis of the acoustic images obtained with the MEMS microphone array using classification techniques based on machine learning, such as Support Vector Machines (SVM).

The tests which have been developed in order to obtain acoustic images of a fan matrix by means of the use of the system based on a planar array of MEMS microphones [11] have been carried out inside an anechoic chamber, as can be observed in Figure 6. Figure 6 shows the assembly of the tests: the array of the system is located opposite the fan matrix to capture the noise that it generates.

It is true that this test must be widened with other tests including background noise and even objects near the assembly in order to create surfaces where the sound generated by the fans could be reflected. These tests would simulate a more real operation situation, because an operative fan matrix is not isolated from the surroundings, nor even placed inside an anechoic chamber.

The hardware placed inside the anechoic chamber is controlled by software developed in LabVIEW. The user interface of this software allows displaying the acoustic signals captured by each of the MEMS microphones of the array, as well as the global acoustic image of the fan matrix, obtained by means of wideband beamforming techniques.

\section{Results and Discussion}

The system shown in previous sections has multiple applications: localization and characterization of noise or vibration sources, spatial filtering and elimination of acoustic interference, and so forth. This case study is focused on obtaining acoustic images of a fan matrix.

The tests carried out with the array of MEMS microphones have allowed the authors to obtain acoustic images of each of the fans that form the fan matrix, as well as acoustic images of the whole fan matrix, that is, with the nine fans working at the same time. Before showing the acoustic images which have been obtained, the acoustic signals received by the microphones of the array have been analyzed in order to understand better the noise generated by the fans.

The acoustic signals obtained by the 64 microphones of the array are the noise that the fans generate. Fan noise is nonstationary or periodic, so the authors decided to capture 1500 signals for each specific test, in order to obtain the averaged signal in each case and analyze it to obtain the averaged behavior of the fans.

Figure 7 shows the averaged FFT spectrum of the noise signals generated by one fan of the matrix. The spectrum has been obtained using the PULSE LabShop v. 13.5.0.32 analysis software platform. Figure 7 shows that the resonance frequency of this is $278 \mathrm{~Hz}$, shown as the highest tone of the spectrum. The rest of the tones shown in Figure 7 are the first, 


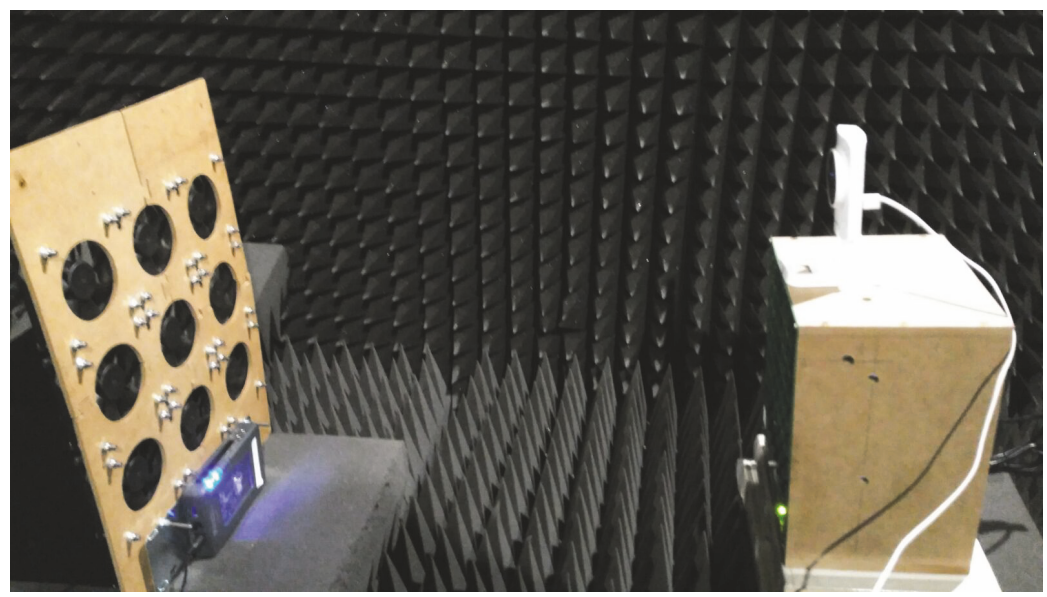

FIgURE 6: Hardware assembly for the tests.

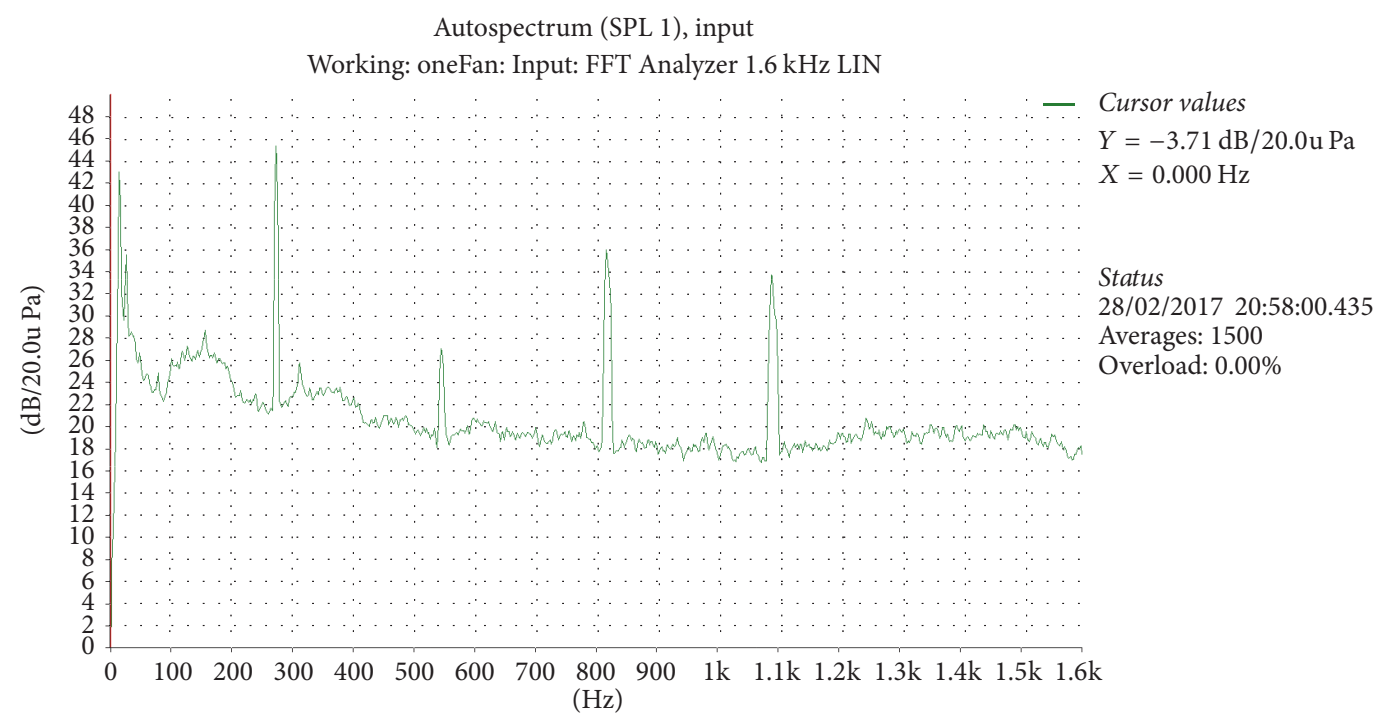

FIGURE 7: Averaged spectrum of the noise generated by one fan.

second, and third harmonics of this resonance frequency: more or less $556 \mathrm{~Hz}, 834 \mathrm{~Hz}$, and $1112 \mathrm{~Hz}$.

Using also a PULSE platform, the averaged FFT spectrum of the noise signals generated by the whole fan matrix has been obtained, and it is shown in Figure 8. This figure shows that the noise behavior of each of the fans of the matrix is not exactly the same. But these different behaviors are very similar to the one shown in Figure 7. In this case, the resonance frequencies vary between 265 and $285 \mathrm{~Hz}$, the first harmonics vary between 530 and $575 \mathrm{~Hz}$, the second harmonics vary between 790 and $860 \mathrm{~Hz}$, and, finally, the third harmonics vary between 1050 and $1150 \mathrm{~Hz}$.

After analyzing these spectra, the acoustic images of each one of the 9 fans of the matrix running alone were obtained using the array of MEMS microphones together with wideband beamforming techniques. For each fan, 1000 acoustic images were generated, in order to obtain 9 consistent averaged acoustic images, which are shown in Figure 9. Analyzing these images, it can be observed that the center of each image seems to be placed in the same position in the matrix where the real fan is.

These centers are the ones of the averaged acoustic images. For each fan, the center of each of the 1000 acoustic images used to obtain the averaged one could not be in the same position as the "averaged center." This effect is shown in Figure 10(a), where it can be observed that the centers of the acoustic images of each fan are scattered around the corresponding "averaged center."

Thanks to the "averaged centers," the positions of the fans in the matrix could be estimated. In Figure 10(b), the "averaged centers" can be compared with the real ones. It can be observed that the estimated positions of the centers are rather near of the real ones. Table 1 shows the errors on the estimated positions of the fans. All errors on the azimuth coordinate are lower than $2.5^{\circ}$, and the mean value of these errors is $1.53^{\circ}$. On the elevation coordinate, errors are even lower; all of them are under $1.5^{\circ}$, and its mean value is $0.81^{\circ}$. So, the averaged acoustic images of the fans could be used to estimate their positions. 


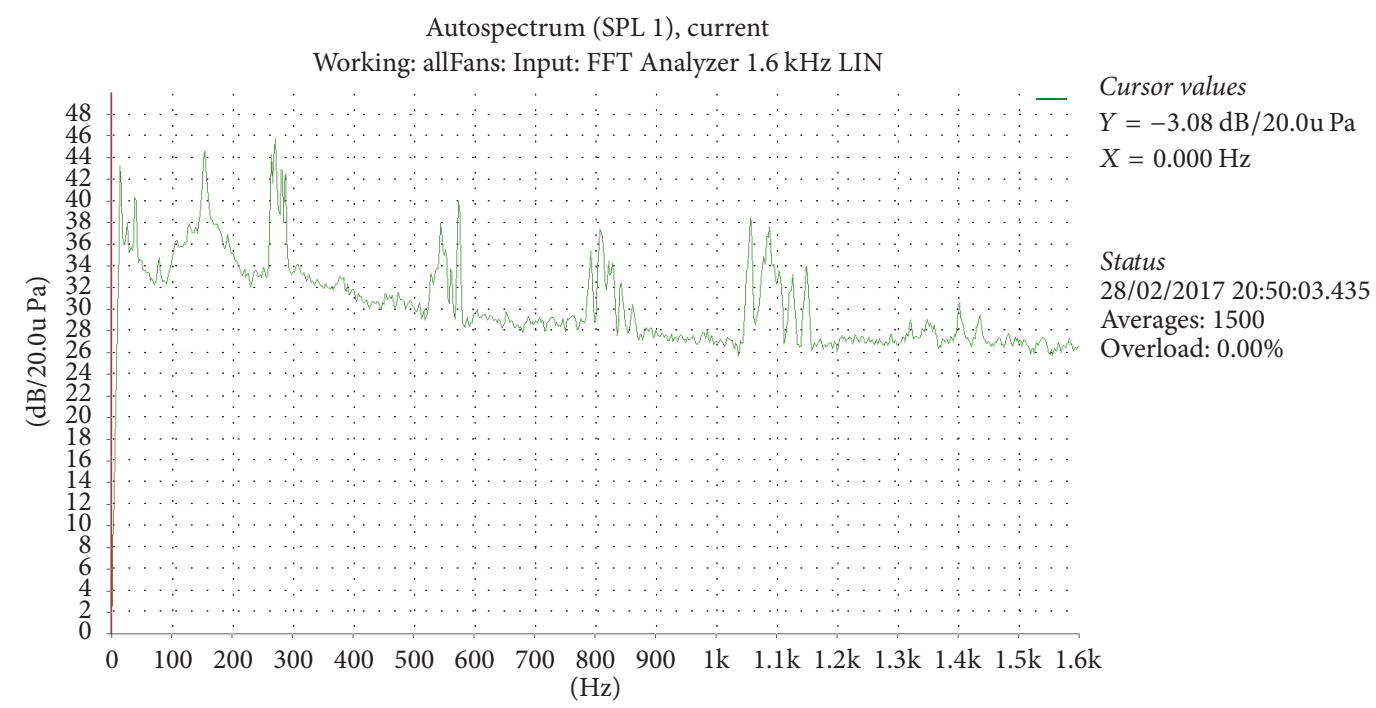

FIGURE 8: Averaged spectrum of the noise generated by the whole fan matrix.

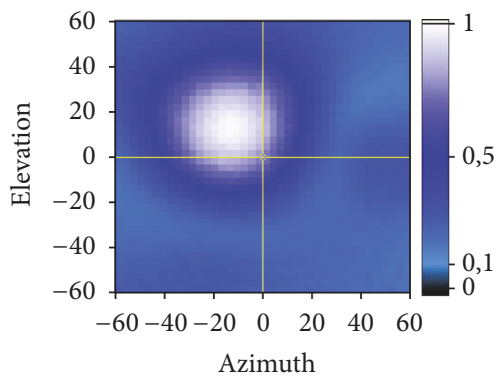

(a)

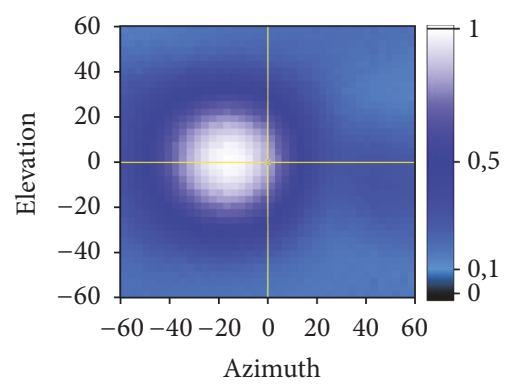

(d)

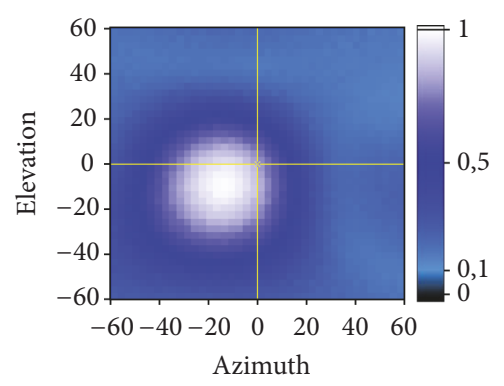

(g)

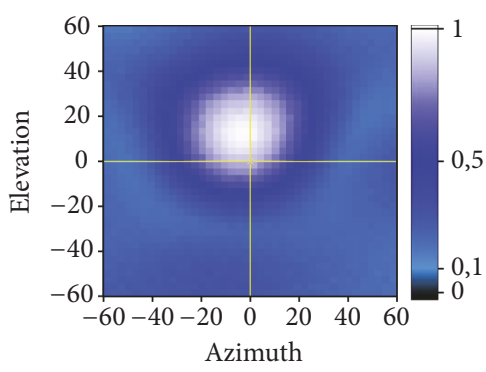

(b)

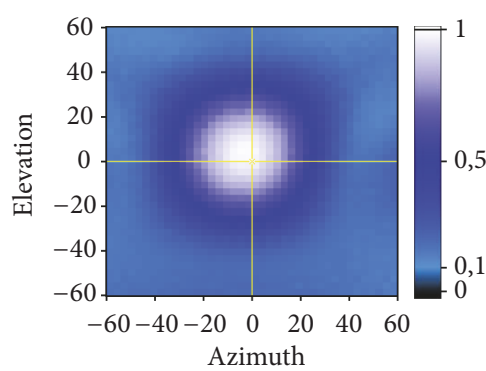

(e)

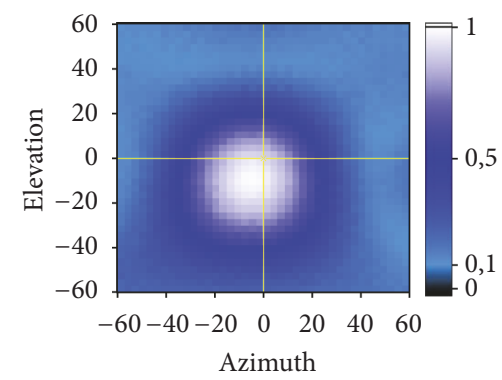

(h)

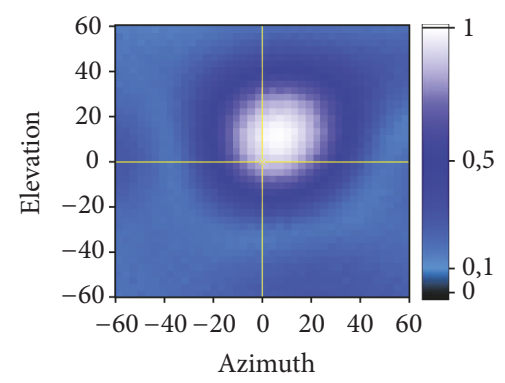

(c)

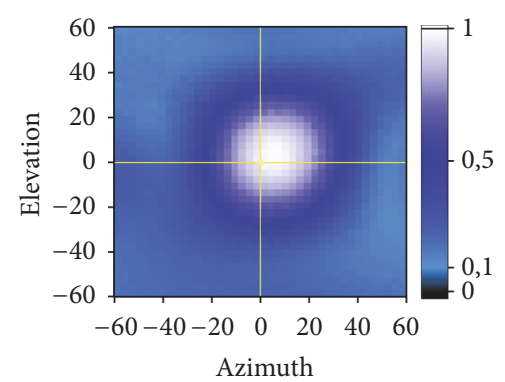

(f)

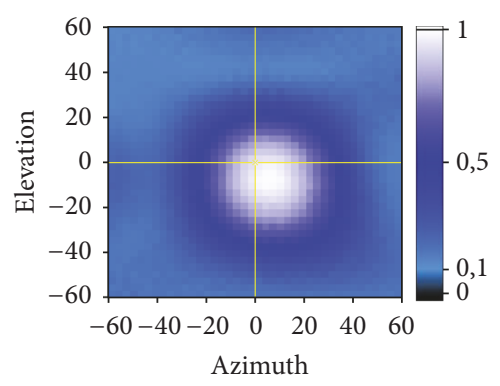

(i)

Figure 9: Acoustic images of each fan of the matrix. 


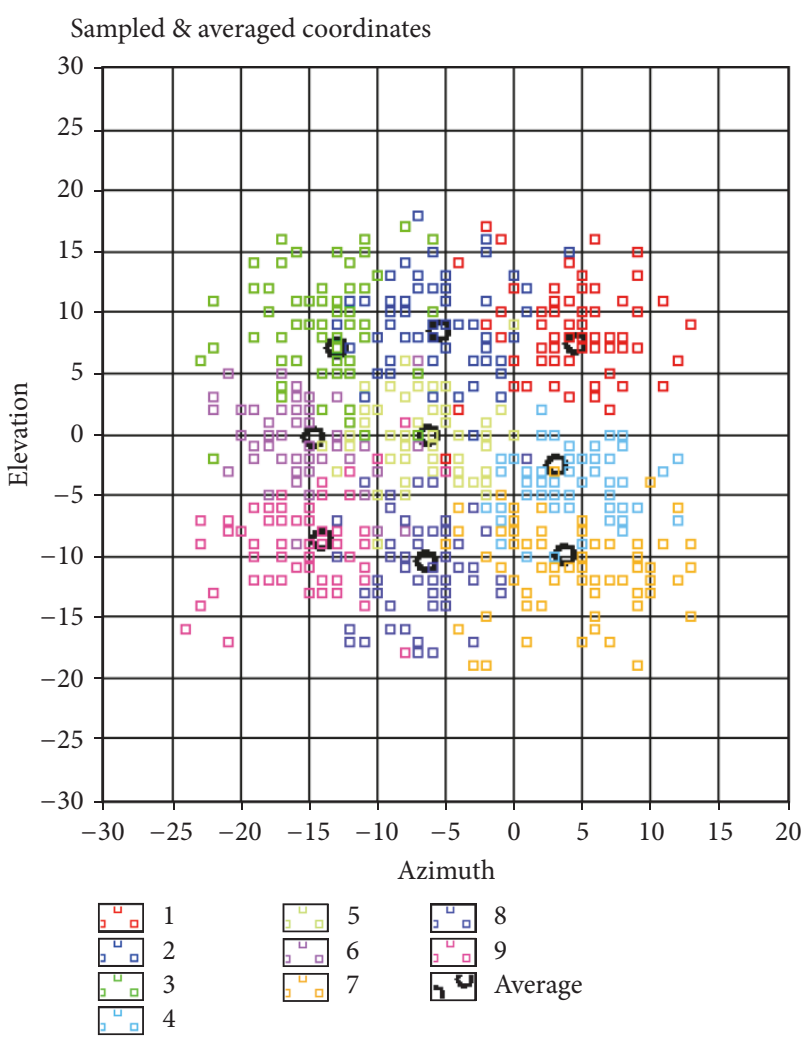

(a)

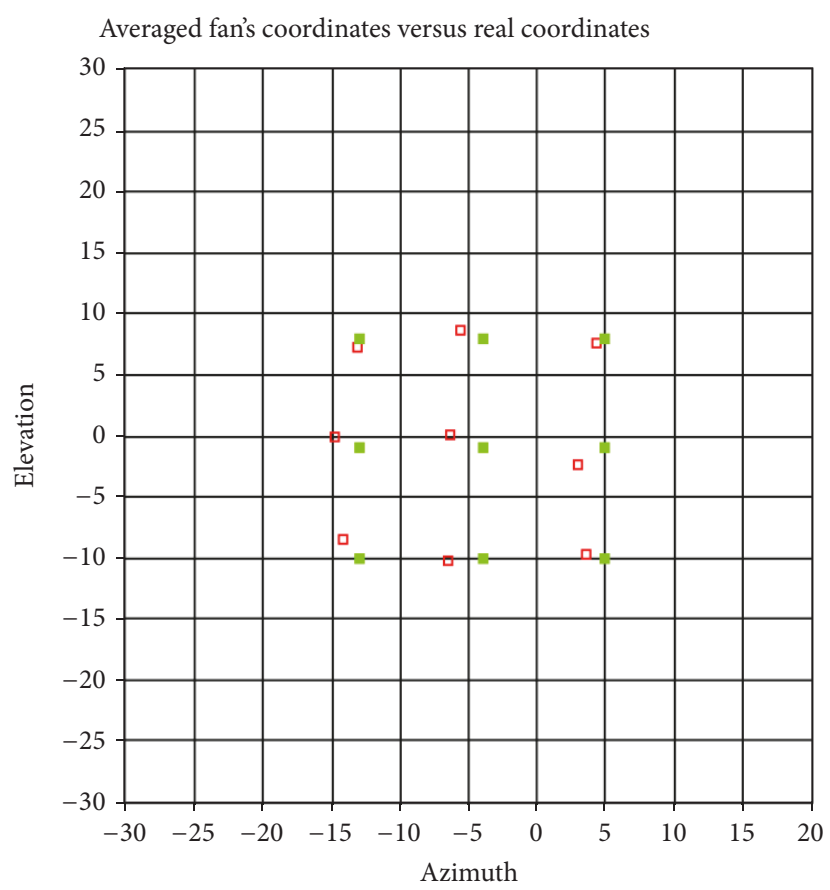

\begin{tabular}{ll}
\hline$\square$ & Real \\
\hline$\square$ & Averaged
\end{tabular}

(b)

Figure 10: (a) Calculated centers of the acoustic images of each fan of the matrix. (b) Real versus estimated center coordinates.

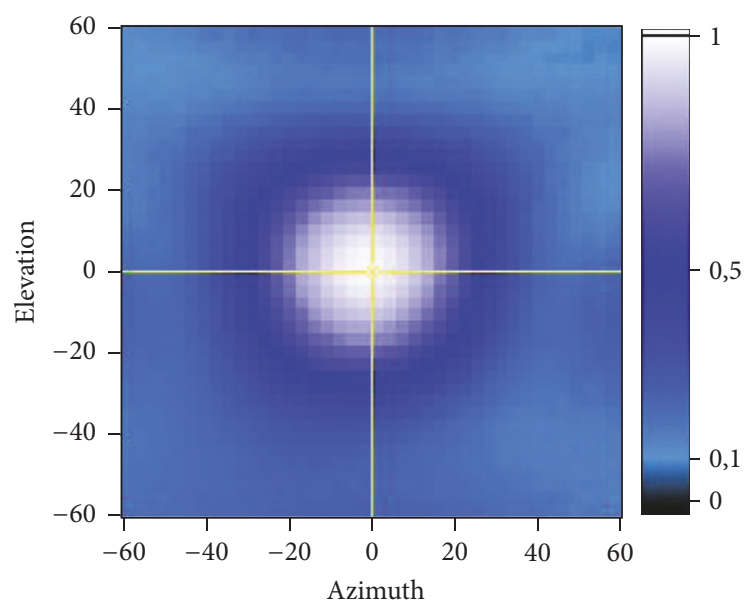

FIGURE 11: Acoustic images of the whole fan matrix.

The last acoustic image that was obtained with the system based on the array of MEMS microphones was the one of the whole fan matrix. This acoustic image is shown in Figure 11. It can be observed that this image seems to be the acoustic image of an only one bigger fan, which is exactly the objective of a fan matrix: several small fans working as a big one in order to produce a certain air flow.
TABLE 1: Errors on the estimated center coordinates.

\begin{tabular}{lcc}
\hline & Azimuth error $\left[^{\circ}\right]$ & Elevation error $\left[^{\circ}\right]$ \\
\hline Fan \#1 & 0.60 & 0.43 \\
Fan \#2 & 1.67 & 0.63 \\
Fan \#3 & 0.15 & 0.77 \\
Fan \#4 & 2.07 & 1.41 \\
Fan \#5 & 2.33 & 1.14 \\
Fan \#6 & 1.83 & 0.92 \\
Fan \#7 & 1.40 & 0.28 \\
Fan \#8 & 2.49 & 0.24 \\
Fan \#9 & 1.25 & 1.46 \\
\hline Averaged error & 1.53 & 0.81 \\
\hline Standard deviation & 1.70 & 0.92 \\
\hline
\end{tabular}

\section{Conclusions}

The tests performed with the system based on the planar array of MEMS microphones, inside an anechoic chamber, have allowed the authors to obtain acoustic images of the working individual fans and of the working whole matrix.

Analyzing the signals received by each MEMS microphone and their corresponding spectra, it was observed that fan noise is nonstationary or periodic, so averaged signals of the acoustic images were calculated in order to estimate the 
averaged behavior of the fans and/or the whole fan matrix. With this analysis it was also observed that although all the fans of the matrix are physically identical, the noise behavior of each of the fans is not exactly the same. At least, the variation of these behaviors is delimited. All the fans have a resonance frequency at around $275( \pm 10) \mathrm{Hz}$ and three harmonics: the first one located at $550( \pm 20) \mathrm{Hz}$, the second one at $825( \pm 35) \mathrm{Hz}$, and the third one at $1100( \pm 50) \mathrm{Hz}$.

Analyzing the corresponding acoustic images of the working individual fans it was observed that the center of each image seems to be placed around the real position of the fan in the matrix. So, the assessment of the center of the averaged acoustic images of the individual fans could be used to estimate the real position of the fan inside the matrix.

Finally, the analysis of the acoustic image of the whole fan matrix reveals the precise objective of the matrix: a bigger acoustic image formed by several small fans working as a bigger one.

So, the results obtained in this study confirm that the acoustic system based on a planar array of MEMS microphones allows the acquisition of acoustic images of a fan matrix, as well as the acoustic images of each fan individually. This work can be considered a previous step of a future study based on fault diagnosis of a fan matrix by means of the analysis of these acoustic images obtained with a MEMS microphone array using classification techniques based on machine learning.

\section{Conflicts of Interest}

The authors declare that there are no conflicts of interest regarding the publication of this paper.

\section{Acknowledgments}

This work has been funded by the Spanish research project SAM (MINECO: TEC 2015-68170-R).

\section{References}

[1] H. Van Trees, Optimum Array Processing: Part IV of Detection, Estimation and Modulation Theory, John Wiley \& Sons, 2002.

[2] M. Brandstein and D. Ward, Microphone arrays, Springer, 2001.

[3] B. D. Van Veen and K. M. Buckley, "Beamforming: a versatile approach to spatial filtering," IEEE ASSP Magazine, vol. 5, no. 2, pp. 4-24, 1988.

[4] L. del Val et al., "Optimisation of sensor positions in random linear arrays based on statistical relations between geometry and performance," Applied Acoustics, vol. 73, no. 1, pp. 78-82, 2012.

[5] J. D. Duran, A. I. Fuente, and J. J. V. Calvo, "Multisensorial modular system of monitoring and tracking with information fusion techniques and neural networks," in Proceedings of IEEE International Carnahan Conference on Security Technology, pp. 59-66, Madrid, Spain, 1999.

[6] A. lzquierdo-Fuente, J. Villacorta-Calvo, M. Raboso-Mateos, A. Martines-Arribas, D. Rodriguez-Merino, and L. del ValPuente, "A human classification system for a video-acoustic detection platform," in Proceedings of the 38th Annual International Carnahan Conference on Security Technology, pp. 145-152, Albuquerque, NM, USA, 2004.
[7] J. Villacorta-Calvo et al., "A Configurable Sensor Network Applied to Ambient Assisted Living," Sensors, vol. 11, no. 11, pp. 10724-10737, 2011.

[8] A. Izquierdo-Fuente, L. Del Val, M. I. Jiménez, and J. J. Villacorta, "Performance evaluation of a biometric system based on acoustic images," Sensors, vol. 11, no. 10, pp. 9499-9519, 2011.

[9] A. I. Fuente, L. D. V. Puente, J. J. V. Calvo, and M. R. Mateos, "Optimization of a biometric system based on acoustic images," The Scientific World Journal, vol. 2014, Article ID 780835, 13 pages, 2014.

[10] L. del Val, A. Izquierdo-Fuente, J. J. Villacorta, and M. Raboso, "Acoustic biometric system based on preprocessing techniques and linear support vector machines," Sensors (Switzerland), vol. 15, no. 6, pp. 14241-14260, 2015.

[11] A. Izquierdo, J. J. Villacorta, L. del Val Puente, and L. Suárez, "Design and evaluation of a scalable and reconfigurable multiplatform system for acoustic imaging," Sensors (Switzerland), vol. 16, no. 10, article 1671, 2016.

[12] W. Siong Gan, Acoustic Imaging: Techniques and Applications for Engineers, John Wiley \& Sons, 2012.

[13] J. Tiete, F. Domínguez, B. da Silva, L. Segers, K. Steenhaut, and A. Touhafi, "SoundCompass: a distributed MEMS microphone array-based sensor for sound source localization," Sensors, vol. 14, no. 2, pp. 1918-1949, 2014.

[14] A. Edstrand et al., "An Aeroacoustic Microelectromechanical Systems (MEMS) Phased Microphone Array," in Proceedings of the 21st AIAA Aerodynamic Decelerator Systems Technology Conference and Seminar, Dublin, Ireland, May 2011.

[15] X. Zhang, E. Song, J. Huang et al., "Acoustic source localization via subspace based method using small aperture MEMS arrays," Journal of Sensors, vol. 2014, Article ID 675726, 14 pages, 2014.

[16] X. Zhang, J. Huang, E. Song, H. Liu, B. Li, and X. Yuan, "Design of small MEMS microphone array systems for direction finding of outdoors moving vehicles," Sensors, vol. 14, no. 3, pp. 43844398, 2014

[17] E. Zwyssig, F. Faubel, S. Renals, and M. Lincoln, "Recognition of overlapping speech using digital MEMS microphone arrays," in Proceedings of the 38th IEEE International Conference on Acoustics, Speech, and Signal Processing (ICASSP '13), pp. 70687072, 2013.

[18] I. Hafizovic, C.-I. C. Nilsen, M. Kjølerbakken, and V. Jahr, "Design and implementation of a MEMS microphone array system for real-time speech acquisition," Applied Acoustics, vol. 73, no. 2, pp. 132-143, 2012.

[19] R. D. White, J. Krause, R. De Jong, G. Holup, J. Gallman, and M. Moeller, "MEMS microphone array on a chip for turbulent boundary layer measurements," in Proceedings of the 50th AIAA Aerospace Sciences Meeting Including the New Horizons Forum and Aerospace Exposition, January 2012.

[20] R. Groschup and C. U. Grosse, "MEMS microphone array sensor for air-coupled impact-echo," Sensors (Switzerland), vol. 15, no. 7, pp. 14932-14945, 2015.

[21] M. Turqueti, J. Saniie, and E. Oruklu, "Scalable acoustic imaging platform using MEMS array," in Proceedings of the IEEE International Conference on Electro/Information Technology (EIT '10), May 2010.

[22] C. Vanwynsberghe, R. Marchiano, F. Ollivier, P. Challande, H. Moingeon, and J. Marchal, "Design and implementation of a multi-octave-band audio camera for realtime diagnosis," Applied Acoustics, vol. 89, pp. 281-287, 2015.

[23] "Sorama: Sound Solutions," https://www.sorama.eu/Solution/ measurements. 
[24] I. Vér and L. Beranek, Noise and Vibration Control Engineering, John Wiley and Sons, 2nd edition, 2006.

[25] K. Chen, J. Si, F. Zhou, R. Zhang, H. Shao, and H. Zhao, "Optimization of air quantity regulation in mine ventilation networks using the improved differential evolution algorithm and critical path method," International Journal of Mining Science and Technology, vol. 25, no. 1, pp. 79-84, 2015.

[26] K. Gao, "Application of mathematical approximation method in improving mine ventilation system with multi-fans," Journal of Liaoning Technical University - Natural Science Edition, vol. 31, no. 6, pp. 834-837, 2012.

[27] N. Staunton, V. Pickert, and R. Maughan, "Assessment of advanced thermal management systems for micro-hybrid trucks and heavy duty diesel vehicles," in Proceedings of the IEEE Vehicle Power and Propulsion Conference (VPPC '08), September 2008.

[28] A. Jagarwal, "Advanced automotive thermal management Operation of an electric radiator fan array for reduced electric power consumption," Thesis. Paper 1015, 2010.

[29] T. T. Wang, A. Jagarwal, J. R. Wagner, and G. Fadel, "Optimization of an Automotive Radiator Fan Array Operation to Reduce Power Consumption," IEEE/ASME Transactions on Mechatronics, vol. 20, no. 5, pp. 2359-2369, 2015.

[30] T. Wang and J. Wagner, "Advanced automotive thermal management - Nonlinear radiator fan matrix control," Control Engineering Practice, vol. 41, pp. 113-123, 2015.

[31] Z. Qi, B. Wang, X. Pei, and G. Ma, "A method of road surface identification based on road characteristics and the features of antilock braking adjustment," Automotive Engineering, vol. 36, no. 3, pp. 552-565, 2014.

[32] T.-J. Wang, X.-P. Xie, J.-G. Li et al., “Optimization of monitoring points' layout for the multi-fan and multi-station ventilation system," Applied Mechanics and Materials, vol. 614, pp. 113-117, 2014.

[33] T. Okayama et al., "A simulation for precision airflow control using multi-fan in a plant factory," Environ. Control Biol, vol. 46, no. 3, pp. 183-194, 2008.

[34] T. Adams and M. Wu, "Case study of a 10-story office building with four 200, 000 CFM fan-wall arrays," in Proceedings of the in Proceedings of the Internoise Conference, San Francisco, California, USA, 2015.

[35] M. Sahini, E. Kumar, T. Gao, C. Ingalz, A. Heydari, and S. Xiaogang, "Study of Air Flow Energy within Data Center room and sizing of hot aisle Containment for an Active vs Passive cooling design," in Proceedings of the 15th InterSociety Conference on Thermal and Thermomechanical Phenomena in Electronic Systems (ITherm '16), pp. 1453-1457, June 2016.

[36] "Engineering a Fan-Wall Solution at the Art Institute of Chicago," https://hpac.com/iaq-amp-ventilation/engineeringfan-wall-solution.

[37] S. Ozono, A. Nishi, and H. Miyagi, "Turbulence generated by a wind tunnel of multi-fan type in uniformly active and quasi-grid modes," Journal of Wind Engineering and Industrial Aerodynamics, vol. 94, no. 4, pp. 225-240, 2006.

[38] M. Noda, M. Ninomiya, and F. Nagao, "Development of a new tornado simulator with multi-fan and multi-vane," Journal of Wind Engineering, vol. 39, no. 1, pp. 13-16, 2014.

[39] P. Huang, "Development of devices and methods for simulation of hurricane winds in a full-scale testing facility," Wind and Structures, vol. 12, no. 2, pp. 151-177, 2009.
[40] T. Baheru, A. Gan Chowdhury, G. Bitsuamlak, F. J. Masters, and A. Tokay, "Simulation of wind-driven rain associated with tropical storms and hurricanes using the 12-fan Wall of Wind," Building and Environment, vol. 76, pp. 18-29, 2014.

[41] A. M. Aly, A. G. Chowdhury, and J. Erwin, "Design and fabrication of a new open jet electric-fan wall of wind facility for coastal research," in Proceedings of the Engineering Mechanics Conference, pp. 137-148, Los Angeles, California, USA.

[42] C. T. Hsieh, J.-M. Ting, C. Yang, and C. K. Chung, "The introduction of MEMS packaging technology," in Proceedings of the 4th International Symposium on Electronic Materials and Packaging (EMAP '02), pp. 300-306, December 2002.

[43] S. Beeby et al., MEMS Mechanical Sensors, Artech House Publishers, 2004.

[44] P. R. Scheeper, A. G. H. van der Donk, W. Olthuis, and P. Bergveld, "A review of silicon microphones," Sensors and Actuators: A. Physical, vol. 44, no. 1, pp. 1-11, 1994.

[45] A. Dehé, "Silicon microphone development and application," Sensors and Actuators, A: Physical, vol. 133, no. 2, pp. 283-287, 2007.

[46] G. Feiertag, M. Winter, and A. Leidl, "Packaging of MEMS microphones," in Proceedings of the Smart Sensors, Actuators, and MEMS IV, May 2009.

[47] "Acoustic System of Array Processing Based on High-Dimensional MEMS Sensors for Biometry and Analysis of Noise and Vibration," http://sine.ni.com/cs/app/doc/p/id/cs-16913.

[48] "Foxconn D90SM-12 3-Pin 92MM Fan," http://www.cwcgroup.com/d90sm12.html. 


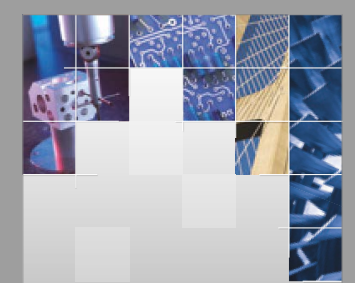

\section{Enfincering}
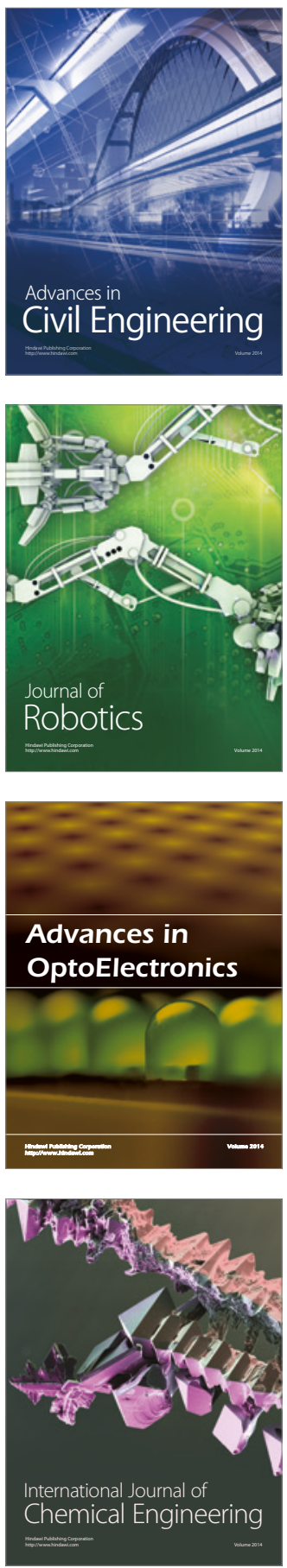

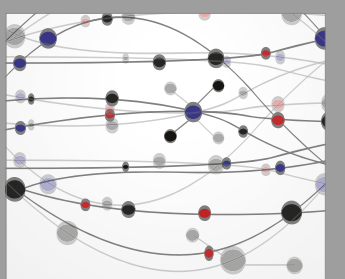

The Scientific World Journal

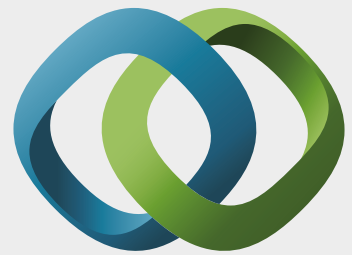

\section{Hindawi}

Submit your manuscripts at

https://www.hindawi.com
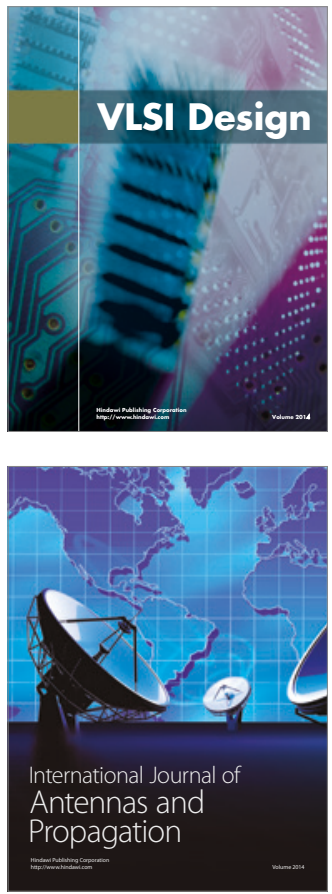

\section{Rotating}

Machinery
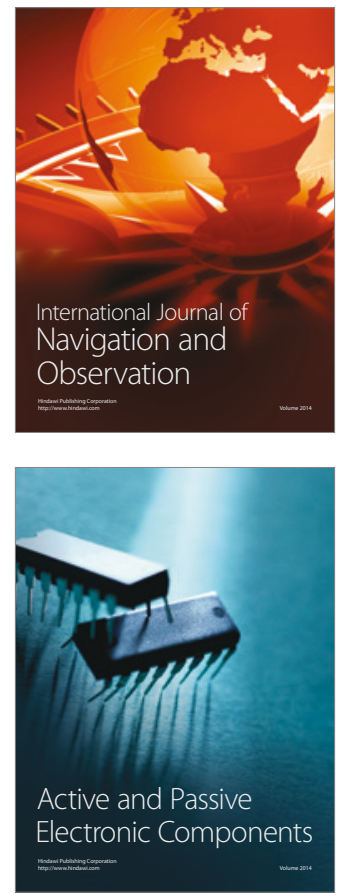
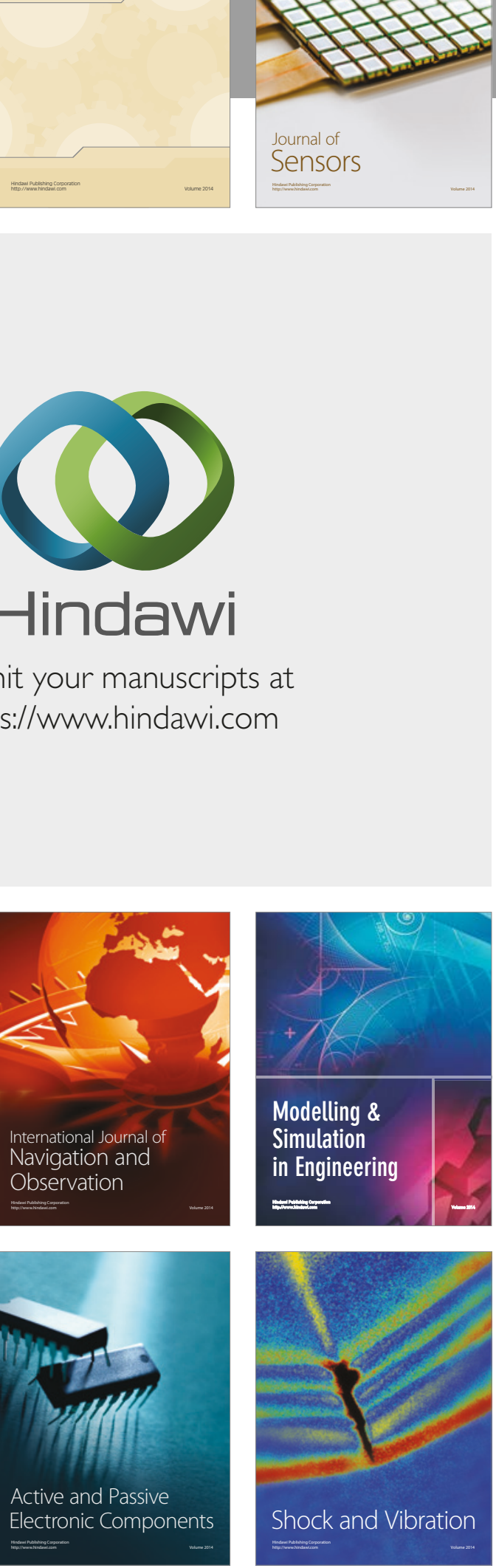
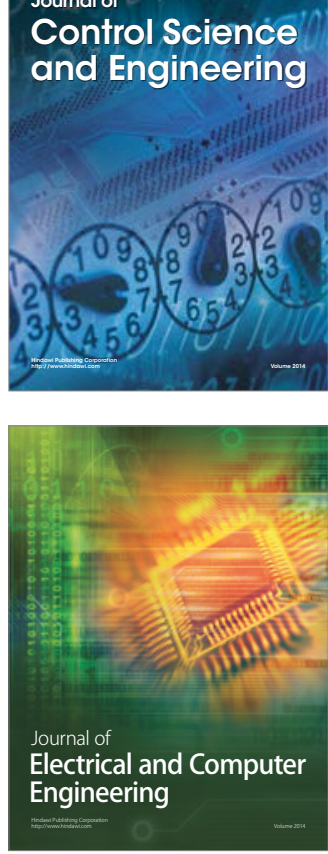

Distributed

Journal of

Control Science

and Engineering
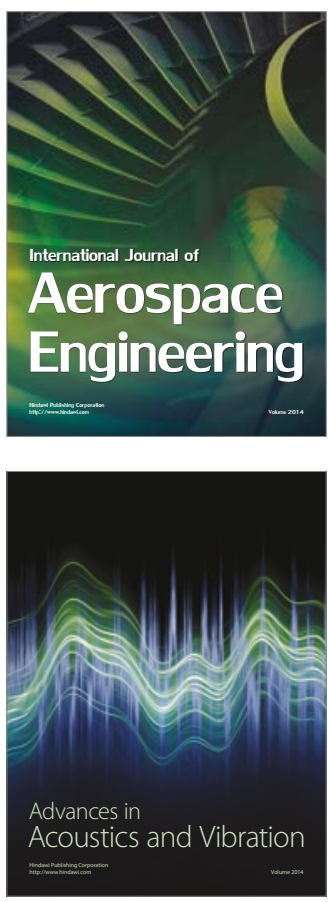

Sensor Networks 